\title{
The relationship between social capital components and control of type 2 diabetes: A path analysis model
}

\author{
Yousef Moradi ${ }^{1}$, Mahshid Nasehi ${ }^{1}$, Mohsen Asadi-Lari ${ }^{1}$, Mohamad Ebrahim Khamseh ${ }^{2}$, \\ Hamid Reza Baradaran²*
}

\begin{abstract}
Background: Social capital is an important interpersonal organizational resource that may affect health behaviors and seems to be an important factor in chronic diseases. Considering the lack of evidence on this topic, in the present study, we aimed at investigating the association between components of social capital, socioeconomic condition, and controlling Type 2 diabetes mellitus in Iran.

Methods: This study was conducted on 300 patients with Type 2 diabetes in Tehran, Iran. In this study, simple random sampling method was used for data collection. Path analysis model was used to examine the potential association between social capital components and to determine the factors that control Type 2 diabetes.

Results: In the present study, most of the participants were female (70.7\%) and married (78.7\%). Path coefficients were calculated by a series of multiple regression analyses based on the conceptual model. The final model had a proper fit with Chi-square $=2.08$ (DF $=1, \mathrm{P}=0.049), \mathrm{GFI}=0.542, \mathrm{AGFI}=0.915, \mathrm{NFI}=0.228, \mathrm{RFI}=0.284, \mathrm{IFI}=0.299, \mathrm{TLI}=0.292, \mathrm{CFI}=0.219$, and $\mathrm{RMSEA}=0.033$. The Groups and networks (network) $(\beta=0.051, p=0.036)$ and trust and solidarity $(\beta=-0.018, p=0.028)$ had a direct positive and negative effect on $\mathrm{HbA1C}$, respectively. The results also revealed that education levels had a direct positive effect on groups and networks (network) $(\beta=0.118, p=0.036)$, trust and solidarity $(\beta=0.082, p=0.007)$, information and communication $(\beta=0.037, p=$ $0.027)$, and contribution in team works and public activities $(\beta=0.064, p=0.003)$. In addition, education levels had an indirect positive effect on HbA1C through their effect on groups and networks (network), trust and solidarity, and information and communication.

Conclusion: Social capital has a significant relationship with diabetes. Although it was not specified clearly which components of social capital were associated with diabetes, trust and solidarity showed a negative direct effect on HbA1C, respectively. Therefore, developing and implementing local and collective programs to build trust and increase the standards of social trust in patients with diabetes can be effective in controlling HbA1C.
\end{abstract}

Keywords: Social capital, Blood glucose, Diabetes, Iran

Copyright@ Iran University of Medical Sciences

Cite this article as: Moradi Y, Nasehi M, Asadi-Lari M, Khamseh ME, Baradaran HR. The relationship between social capital components and control of type 2 diabetes: A path analysis model. Med J Islam Repub Iran. 2017 (14 Mar);31:21. https://doi.org/10.18869/mjiri.31.21

\section{Introduction}

Diabetes is the most common metabolic disease, with a dramatically rising prevalence worldwide (1-2). It is estimated that by the year 2030 the number of people with diabetes reach over 336 million, more than 2 times the value of the year 2000 (3). Diabetes affects all aspects of a patient's life, and its control requires a fundamental change in lifestyle, also, diabetes is one of the most important public health challenges to both developing and developed countries (3).

Social capital is a means of communication that leads to mutual trust and improved quality of life. Moreover, so-

Corresponding author: Dr Hamid Reza Baradaran, baradaran.hr@iums.ac.ir

1. Department of Epidemiology, School of Public Health, Iran University of Medical Sciences, Tehran, Iran.

2. Endocrine Research Center, Institute of Endocrinology and Metabolism, Iran University of Medical Sciences, Tehran, Iran. cial capital is defined as features of social organization such as trust, norms, and networks that can improve the efficacy of the society by smoothing the way for coordinated actions (4). Social capital affects human health through the following approaches: diffusing knowledge on health promotion, maintaining healthy behavior, accessing health care services, acquiring emotional or material support, and maintaining mutual respect in social networks (5-7). Despite significant improvements in controlling diabetes, the growing trend of this disease has not yet been stopped, and to do so, identifying the social de-

$\uparrow$ What is "already known" in this topic:

Different studies have tried to explore the relationship between social capital and chronic diseases such as diabetes.

\section{$\rightarrow$ What this article adds:}

Increase in social capital and its related components in patients with type 2 diabetes can result in lower HbA1C levels. 
terminants of diabetes are of great importance (8-11). Nowadays, the concept of health is tied with social factors, and many physical and mental diseases greatly correspond to social factors. In recent years, we have witnessed introduction of the term "social capital" in the realm of science, social sciences, economics, and health sciences(12-13). Social capital, as a new concept alongside other forms of capital, stands at an equal level of importance, and is identified by components such as trust, collaboration, correlation, assistance, social support, cooperation, and one-heartedness. Thus, social relations and symbiosis of people in a society are the paragons of identifying social capital (14). Potential mechanisms by which social capital has an impact on health include an increase in health behavior, health improvement, exercise, better access to services, increased healthcare, and reduction in violence and crime among neighbors. Awareness about the impact of social capital components on health and self-care will be of great value in designing interventions for diabetes care in the future. The present study aimed at examining the association between components of social capital and controlling Type 2 diabetes in the Iranian culture.

\section{Methods}

Using a random sampling method, we conducted a crosssectional study on patients with Type 2 diabetes who referred to the diabetic clinic at the Institute of Endocrinology and Metabolism, affiliated to Iran University of Medical Sciences, Tehran, Iran, from August to October 2015. In this study, 300 patients with Type 2 diabetes mellitus, who referred to the clinic, were consecutively enrolled.

Inclusion criteria included patients diagnosed with Type 2 diabetes, aged 30 years or older, and not being hospitalized. Social capital was measured by a valid and reliable questionnaire developed and deigned by Nedjat et al. (15), with 6 dimensions, which are as follow: groups and networks, trust and solidarity, collective action and coopera- tion, information and communication, social cohesion and inclusion, and empowerment and political action. A trained interviewer asked patients to fill in the Social Capital Questionnaire, which took 30 minutes to complete on average. In addition to the demographic variables, the interviewer extracted complications of diabetes and $\mathrm{HbA} 1 \mathrm{C}$ from patients' medical files.

Ethical approval for the study was obtained from the Ethics Committee of Iran University of Medical Sciences. Written consent forms were obtained from the participants, and for those patients who were illiterate and/or unable to read, the consent form was read by the researcher, and then, verbal consent was obtained.

\section{Statistical Analysis}

Cronbach's alpha values were calculated and used to evaluate the reliability of the social capital scale in the reliability analysis.

In the present study, path analysis was used for statistical analysis of $\mathrm{HbA1C}$ as the dependent variable in the model. We considered age, duration of disease, education level, and location as independent variables, and Hbalc level as a dependent variable. Social capital components were considered as mediator variables in the conceptual model (Figure 1).

Factors affecting HbA1C levels have been observed using the fit model demonstrated in Figure 2. The path model was used to examine the direct and indirect relationships among the variables. Goodness-of-fit of the final model was assessed using Chi-square test and the goodness- of- fit indices such as root mean square error of approximation (RMSEA), goodness-of-fit index (GFI), adjusted goodnessof-fit index (AGFI), normed fit index (NFI), relative fit index (RFI), incremental fit index (IFI), Tacker- Lewis index (TLI), and comparative fit index (CFI). Values for GFI, AGFI, NFI, RFI, IFI, TLI, and CFI range from 0 to 1 , with values greater than 0.90 indicating a good fit. Conventionally, there is a good fit if RMSEA is less than 0.05 , and

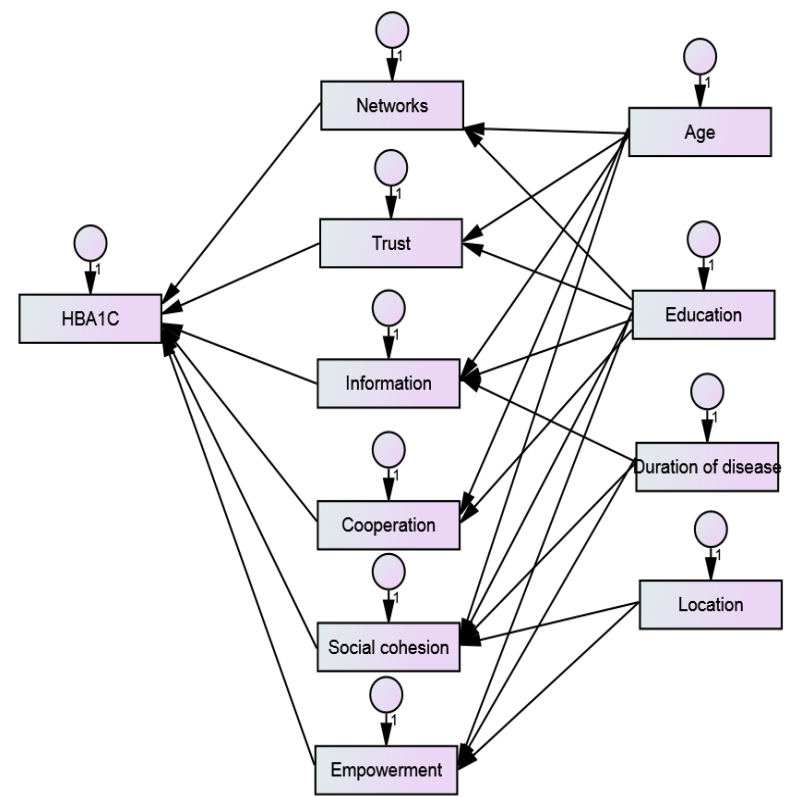

Fig. 1. Conceptual model for pathway analysis of effective factors on HbA1C 


\begin{tabular}{lll} 
Table 1. Characteristics of the Study Population & \\
\hline Variable & Category & Descriptive \\
\hline Age (Year) & - & $43.05 \pm 6.8$ \\
Duration of diabetes (Year) & - & $9.75 \pm 6.1$ \\
HbA1C value & - & $8.17 \pm 2.0$ \\
Gender & Female & $212(70.7 \%)$ \\
& Male & $88(29.3 \%)$ \\
Marital status & Single & $30(10 \%)$ \\
& Married & $236(78.7 \%)$ \\
Education level & Widow/ Divorced & $38(11.4 \%)$ \\
& Illiterate & $62(20.7 \%)$ \\
& Elementary school & $65(21.7 \%)$ \\
Treatment type & High school & $117(39.0 \%)$ \\
& Academic & $55(18.3 \%)$ \\
& Oral hypoglycemic agents & $159(53.0 \%)$ \\
Place of living & Insulin & $91(30.3 \%)$ \\
& Oral hypoglycemic agents + insulin & $47(15.7 \%)$ \\
& No drug use & $3(1 \%)$ \\
& Rural & $67(22.3 \%)$ \\
& Urban & $233(77.7 \%)$ \\
\hline
\end{tabular}

there is adequate fit if RMSEA is less than 0.08 .

\section{Results}

Most participants were female $(70.7 \%)$ and married (78.7\%). Less than half of the respondents did not have complications, but some respondents had diabetes complications $(52.6 \%)$ including cardiovascular diseases, retinopathy, nephropathy, and neuropathy. The average age of the patients was 43 years (The average age was 45 in patients with complication, and in those without complication it was 40 years.). Mean $\pm \mathrm{SD}$ duration of disease in patients with complication was $12.78 \pm 6.26$ years, and in patients with no complication was $6.42 \pm 3.83$ years. The mean (SD) HbA1C levels in patients with complication and no complications were $8.40( \pm 2.03)$ and $6.42(3.83)$ milligram per deciliter, respectively (Table 1 ).

The overall Cronbach's alpha coefficient for components of social capital was 0.75 . Cronbach's alpha coefficient for each dimension was as follows: groups and networks: 0.82 ; trust and solidarity: 0.89 ; collective action and cooperation: 0.72; information and communication: 0.60; empowerment and political action: 0.61 ; and social cohesion and inclusion: 0.91 .

Path coefficients were calculated by a series of multiple regression analyses based on the conceptual model (Figure 1). The final model (Figure 2) had a proper fit with Chisquare $=2.08(\mathrm{DF}=1, \mathrm{P}=0.049), \mathrm{GFI}=0.542, \mathrm{AGFI}=$ $0.915, \mathrm{NFI}=0.228, \mathrm{RFI}=0.284, \mathrm{IFI}=0.299, \mathrm{TLI}=0.292$, $\mathrm{CFI}=0.219$, and RMSEA $=0.033$. The Groups and net- works (network) $(\beta=0.051, \mathrm{p}=0.036)$ and trust and solidarity $(\beta=-0.018, p=0.028)$ had direct positive and negative effects on $\mathrm{HbA1C}$. The results also revealed that education levels had direct positive effects on groups and networks (network) $(\beta=0.118, \mathrm{p}=0.036)$, trust and solidarity $(\beta=0.082, p=0.007)$, information and communication $(\beta$ $=0.037, \mathrm{p}=0.027)$, and contribution in team works and public activities $(\beta=0.064, p=0.003)$. In addition, education levels had indirect positive effects on $\mathrm{HbA} 1 \mathrm{C}$ through their effect on groups and networks (network), trust and solidarity, and information and communication. Modeling results are presented in Tables 2 and 3.

\section{Discussion}

In line with our findings, Crosby et al., in their study, found that social capital indices have a significant relationship with diabetes; however, it was not specified which social capital components were associated with diabetes (16). In another study conducted by Long et al., they revealed a significant relationship between the teamwork component of social capital and blood glucose control. This is while no significant relationship was detected between "participation in organizations" and "truthfulness and honesty among neighbors" components (11).

Our findings suggest that patients with Type 2 diabetes, who had higher levels of groups and networks, showed increased HbA1C levels. There is no clear justification for this finding, however, the possible explanation could be that when patients participate in various groups, they expe-

Table 2. Path coefficient factors on social capital components

\begin{tabular}{|c|c|c|c|c|c|c|c|}
\hline \multicolumn{2}{|c|}{ Social Capital Components } & Association & Variables & Estimate & S.E. & C.R. & $\mathrm{P}$ value \\
\hline \multicolumn{2}{|c|}{ Membership in associations and groups } & $<--$ & Education & 0.118 & 0.056 & 2.093 & 0.036 \\
\hline \multicolumn{2}{|c|}{ Social trust } & $<--$ & Education & 0.082 & 0.030 & 2.708 & 0.007 \\
\hline \multicolumn{2}{|c|}{ Information and communication } & $<--$ & Education & 0.037 & 0.017 & 2.211 & 0.027 \\
\hline \multicolumn{2}{|c|}{ Contribution in team works } & $<--$ & Education & 0.064 & 0.021 & 3.014 & 0.003 \\
\hline \multicolumn{8}{|c|}{ S.E: Standard Error, C.R: Estimate / S.E } \\
\hline \multicolumn{8}{|c|}{ Table 3. Path coefficient factors on $\mathrm{HbA1C}$} \\
\hline Variables & Association $\quad \mathrm{Sc}$ & \multicolumn{2}{|c|}{ Social Capital Components } & Estimate & S.E. & C.R. & $\mathrm{P}$ value \\
\hline $\mathrm{HbA1C}$ & Infor & \multicolumn{2}{|c|}{ Information and communication } & 1.000 & & & \\
\hline $\mathrm{HbA1C}$ & Member & \multicolumn{2}{|c|}{ Membership in associations and groups } & 0.051 & 0.025 & 2.095 & 0.036 \\
\hline $\mathrm{HbA1C}$ & $<--$ & \multicolumn{2}{|c|}{ Social trust } & -0.018 & 0.046 & -0.388 & 0.028 \\
\hline
\end{tabular}


cial capital should conform to various cultures.

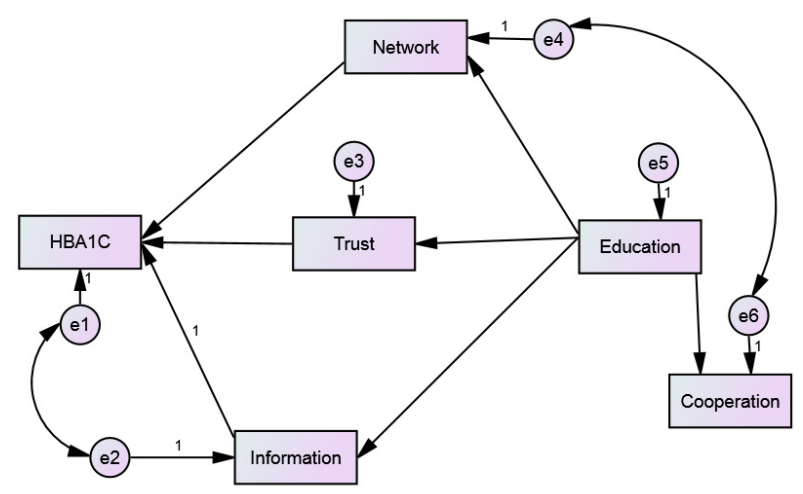

Fig. 2. Final model for Pathway analysis of effective factors on $\mathrm{HbA} 1 \mathrm{C}$

rience stress because of comparing themselves to others and being exposed to overwhelming information, and this stress could lead to uncontrolled HbA1C and complications.

Results have revealed that trust and solidarity induce higher protection rates for controlling Type 2 diabetes. Thus, trust and solidarity, social connection with family, and neighborhoods and labor environment appear to be important factors in controlling diabetes and are protective factors against complications of diabetes. Previous studies have shown that higher levels of family and neighborhood social trust were associated with better control of glycemic levels (17-18). In 2008, Heydari et al. found a significant association between family support and controlling blood glucose levels (19). Moreover, patients who received more support from the family network, managed their blood glucose levels easier $(17,20)$. Findings of the present and similar studies state that increase in social capital indices and its related components in diabetic patients can result in lower HbA1C levels. In clinical terms, lower HbA1C levels reduce macrocellular and microcellular complications of the disease (Each 1\% HbA1C reduction accounts for $37 \%$ reduction in complications.) (21), increase isocial capital components in patients with diabetes, and lead to better control of blood glucose levels. Other studies report that in patients with diabetes, a well-established family, social network, and communication significantly increase the patients' perception about their individual capacity to prevent and manage blood glucose level (22-24). The relationship and communication with family and friends provides a great sense of emotional wellbeing and better decision- making about controlling diabetes (22). Furthermore, communication and information can regulate patients' activities and enable them to comply with treatment, and this will have positive effects on disease control(24). In a qualitative study conducted by Ponsirpongse in Thailand, the findings showed that the "cooperation and membership" component in the diabetes group played a protective role in controlling diabetes (25-27).

\section{Limitations}

One limitation of this study was the way by which social capital was measured. Social capital is influenced by culture and ethnicity, so the instruments used to measure so-

\section{Conclusions}

Social capital has a significant relationship with diabetes. Although it was not specified clearly which social capital components were associated with diabetes, trust and solidarity showed direct and negative effects on $\mathrm{HbA} 1 \mathrm{C}$, respectively. Therefore, local and collective programs should be designed and implemented to build trust and increase standards of social trust in patients with diabetes to empower them to effectively control their HbAlC.

\section{Acknowledgements}

Authors acknowledged all patients for their participation.

Conflict of Interests: The authors declare that they have no competing interests.

\section{References}

1. Chen L, Magliano DJ, Zimmet PZ. The worldwide epidemiology of type 2 diabetes mellitus - present and future perspectives. Nat Rev. 2012;8(4):228-36.

2. Dunstan DW, Zimmet PZ, Welborn TA, De Courten MP, Cameron AJ, Sicree RA, et al. The rising prevalence of diabetes and impaired glucose tolerance; the Australian diabetes, obesity and lifestyle study. Diabetes Care. 2002;25(5):829-34.

3. Danaei G, Finucane MM, Lu Y, Singh GM, Cowan MJ, Paciorek CJ, et al. National, regional, and global trends in fasting plasma glucose and diabetes prevalence since 1980: systematic analysis of health examination surveys and epidemiological studies with 370 country-years and 2.7 million participants. Lancet. 2011;378(9785):31-40.

4. Nyquist F, Forsman AK, Giuntoli G, Cattan M. Social capital as a resource for mental well-being in older people: A systematic review. Aging Ment Health. 2013;17(4):394-410.

5. Glasgow RE, Toobert DJ. Social environment and regimen adherence among type II diabetic patients. Diabetes Care. 1988;11(5):377-86.

6. Blom L, Dahlquist G, Nyström L, Sandström A, Wall S. The Swedish childhood diabetes study - social and perinatal determinants for diabetes in childhood. Diabetologia. 1989;32(1):7-13.

7. Murayama H, Fujiwara Y, Kawachi I. Social capital and health: a review of prospective multilevel studies. J Epid. 2012;22(3):179-87.

8. Schulz AJ, Zenk S, Odoms-Young A, Hollis-Neely T, Nwankwo R, Lockett $\mathrm{M}$, et al. Healthy eating and exercising to reduce diabetes: exploring the potential of social determinants of health frameworks within the context of community-based participatory diabetes prevention. Am J Pub Health. 2005;95(4):645-51.

9. Raphael D, Anstice S, Raine K, McGannon KR, Kamil Rizvi S, Yu V. The social determinants of the incidence and management of type 2 diabetes mellitus: are we prepared to rethink our questions and redirect our research activities? Leader Health Serv. 2003;16(3):10-20.

10. Unwin N, Whiting D, Roglic G. Social determinants of diabetes and challenges of prevention. The Lancet. 2010;375(9733):2204-5.

11. Long JA, Field S, Armstrong K, Chang VW, Metlay JP. Social capital and glucose control. J Commun Health. 2010;35(5):519-26.

12. Betancourt RM, Degnan KO, Long JA. Racial differences in glucose control among patients with type 2 diabetes: a survey on dietary temptations, coping, and trust in physicians. Ethnicity Dis. 2015;23(4):40914.

13. Bigdeli M, Jacobs B, Men CR, Nilsen K, Van Damme W, Dujardin B. Access to Treatment for Diabetes and Hypertension in Rural Cambodia: Performance of Existing Social Health Protection Schemes. PloS one. 2016;11(1).

14. Low LL, Tong SF, Low WY. Social influences of help-seeking behaviour among patients with Type 2 diabetes mellitus in Malaysia. Asia-Pacific Journal of Public Health. 2016;28(1 suppl):17S-25S.

15. Nedjat S, Majdzadeh R, Kheiltash A, Jamshidi E, Yazdani S. Social capital in association with socioeconomic variables in Iran. Soc Indicat Res. 2013;113(3):1153-70.

16. Holtgrave DR, Crosby R. Is social capital a protective factor against 
obesity and diabetes? Findings from an exploratory study. Ann Epidemiol. 2006;16(5):406-8.

17. Mayberry LS, Osborn CY. Family support, medication adherence, and glycemic control among adults with type 2 diabetes. Diabetes Care. 2012;35(6):1239-45.

18. Group UPDS. Effect of intensive blood-glucose control with metformin on complications in overweight patients with type 2 diabetes (UKPDS 34). Lancet. 1998;352(9131):854-65.

19. Heidari SH NM, Hoseini F, Inanlo M, Golgiri F, Shirazi F. Geriatric family support and diabetic type-2 glycemic control. Salmand Iran J Ageing. 2008.

20. Inzucchi SE, Bergenstal RM, Buse JB, Diamant M, Ferrannini E, Nauck M, et al. Management of hyperglycemia in type 2 diabetes: a patient-centered approach position statement of the American Diabetes Association (ADA) and the European Association for the Study of Diabetes (EASD). Diabetes Care. 2012;35(6):1364-79.

21. Alwin DF, Hauser RM. The decomposition of effects in path analysis. Am Sociolog Rev. 1975:37-47.

22. Javanbakht M, Abolhasani F, Mashayekhi A, Baradaran HR. Health related quality of life in patients with type 2 diabetes mellitus in Iran: a national survey. PLoS One. 2012;7(8):e44526.

23. Kennedy A, Rogers A, Vassilev I, Todorova E, Roukova P, Foss C, et al. Dynamics and nature of support in the personal networks of people with type 2 diabetes living in Europe: qualitative analysis of network properties. Health Expectations. 2015;18(6):3172-85.

24. Lins KV, Servaes H, Tamayo A. Social capital, trust, and firm performance during the financial crisis. Trust, and Firm Performance During the Financial Crisis (January 26, 2015). 2015.

25. Hu F, Hu B, Chen R, Ma Y, Niu L, Qin X, et al. A systematic review of social capital and chronic non-communicable diseases. BioScience Trends. 2014;8(6):290-6.

26. Beaglehole R, Bonita R, Horton R, Adams C, Alleyne G, Asaria P, et al. Priority actions for the non-communicable disease crisis. Lancet. 2011;377(9775):1438-47

27. Schmidt MI, Duncan BB, e Silva GA, Menezes AM, Monteiro CA, Barreto SM, et al. Chronic non-communicable diseases in Brazil: burden and current challenges. The Lancet. 2011;377(9781):1949-61. 